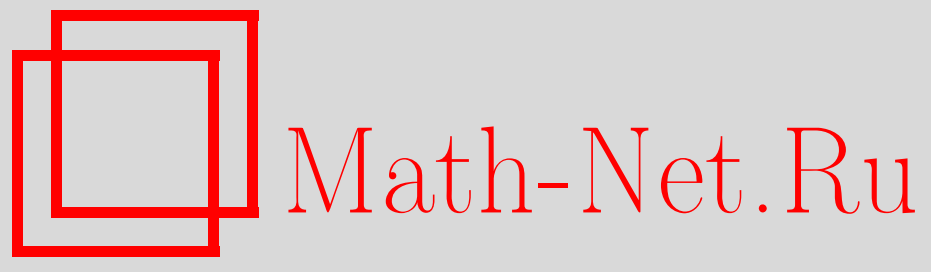

А. В. Михалёв, А. В. Шокуров, Оптимальное использование вейвлет-компонент, УМH, 2007, том 62, выпуск 4, 171-172

DOI: https://doi.org/10.4213/rm7007

Использование Общероссийского математического портала Math-Net.Ru подразумевает, что вы прочитали и согласны с пользовательским соглашением http://www . mathnet.ru/rus/agreement

Параметры загрузки:

IP : 3.89 .197 .203

26 апреля 2023 г., 14:37:38

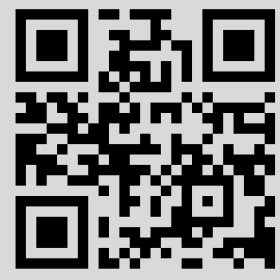




\section{Оптимальное использование вейвлет-компонент}

\section{А. В. Михалёв, А. В. Шокуров}

1. Введение. Рассмотрим линейное преобразование из $\mathbb{R}^{n}$ в $\mathbb{R}^{m}$. Пусть $v \in \mathbb{R}^{n}$, $u \in \mathbb{R}^{m}$ и $u=A v$, где $A$ - матрица линейного преобразования в базисе пространств $\mathbb{R}^{n}$ и $\mathbb{R}^{m}$. Допустим, что нам нужно вычислить не весь вектор $u$, а только его часть. Зная, какие именно компоненты $u$ надо вычислить, можно сократить и вектор $v$. Поэтому интересен вопрос о нахождении минимальной части вектора $v$, по которой можно вычислить необходимую часть вектора $u$.

В статье рассматривается сначала общий случай линейного преобразования, а затем конкретное линейное преобразование, используемое на практике, а именно обратное биортогональное вейвлет-преобразование [1].

2. Линейные преобразования. Пусть $u \in \mathbb{R}^{m}, v \in \mathbb{R}^{n}$ и $u=A v$. Для удобства изложения нумерация компонент векторов и матриц начинается с 0 . При линейном преобразовании компонента $u_{i}$ определяется следующим образом:

$$
u_{i}=\sum_{0 \leqslant k \leqslant n-1} a_{i, k} v_{k}=\sum_{\substack{0 \leqslant k \leqslant n-1 \\ a_{i, k} \neq 0}} a_{i, k} v_{k} .
$$

Поэтому для вычисления компоненты $u_{i}$ нужны только те компоненты $v_{k}$ вектора $v$, которые умножаются на ненулевые компоненты $a_{i, k}$ матрицы $A$. Обозначим через $\operatorname{SupRows}(A,\{i\})$ соответствующее множество индексов: $\operatorname{SupRows}(A,\{i\})=\left\{k: a_{i, k} \neq 0\right\}$. Если, помимо компоненты $u_{i_{1}}$, необходимо вычислить и компоненты $u_{i_{2}}, \ldots, u_{i_{l}}$, то надо взять объединение всех $\operatorname{SupRows}\left(A,\left\{i_{p}\right\}\right)$, а именно: $\operatorname{SupRows}\left(A,\left\{i_{1}, \ldots, i_{l}\right\}\right)=$ $\bigcup_{p=1} \operatorname{SupRows}\left(A,\left\{i_{p}\right\}\right)$.

3. Случай свертки. По вектору $v \in \mathbb{R}^{n}$ построим симметрично-периодический вектор $v_{\{\cdot\}}$ значимой длины $n$, определив его компоненты для произвольного целого числа $i$ следующим образом:

$$
v_{\{i\}}=\left\{\begin{array}{lrl}
v_{i}, & i & \in[0, n-1] ; \\
v_{-i}, & -i & \in[0, n-1] ; \\
v_{\{i-k \cdot \text { len }\}}, & i & \in[1-n-k \cdot \text { len }, n-1-k \cdot \text { len }]
\end{array}\right.
$$

где len $=2 n-1-$ его период. Свертка вектора $v_{\{\cdot\}}$ с ядром $h \in \mathbb{R}^{\mathbb{Z}}$ есть вектор $u \in \mathbb{R}^{n}$ с компонентами

$$
u_{p}=\left(h \star v_{\{\cdot\}}\right)_{p}=\sum_{n=-\infty}^{+\infty} h_{p-n} v_{\{n\}} .
$$

Свертка линейна и может быть задана матрицей $A$, при этом преобразование $(1)$ записывается в виде $u=A v$.

В настоящей заметке всюду предполагается, что ядро $h$ сосредоточено в окрестности нуля радиуса меньше $n$. Тогда существуют такие целые числа $l, r \in \mathbb{Z}, l \leqslant 0$, $r \geqslant 0,\|l\|,\|r\|<n$, что при $i<l$ и при $i>r$ выполнено равенство $h_{i}=0$. Иначе считаем, что $\operatorname{SupRows}(A,\{i\})=\{0, \ldots, n-1\}$.

Найдем явный вид матрицы $A$.

Рассмотрим сначала случай, когда $h$ обращается в нуль при отрицательных индексах, т. е. $l=0, r \geqslant 0$. В этом случае получаем матрицу $A^{+}$. Индекс первого ненулевого элемента в строчке $i$ и индекс последнего ненулевого элемента в строчке $j$ матрицы $A^{+}$соответственно равны

$$
S\left(A^{+}, i\right)=\left\{\begin{array}{ll}
0, & i \leqslant r, \\
i-r, & i>r,
\end{array} \quad E\left(A^{+}, j\right)= \begin{cases}r-j, & 2 j \leqslant r \\
j, & 2 j>r .\end{cases}\right.
$$


Случай, когда $h$ равно нулю при положительных индексах, рассматривается аналогичным образом.

Обозначим $\overrightarrow{i-j}=\{i, i+1, \ldots, j-1, j\}$. В итоге получаем следующую формулу:

$$
\operatorname{SupRows}(A, \overrightarrow{i-j})=\left\{i_{\text {start }}, i_{\text {start }+1}, \ldots, i_{\text {end }-1}, i_{\text {end }}\right\},
$$

где $\operatorname{start}=\min \left(S\left(A^{-}, \overrightarrow{i-j}\right), S\left(A^{+}, \overrightarrow{i-j}\right)\right)$, end $=\max \left(E\left(A^{-}, \overrightarrow{i-j}\right), E\left(A^{+}, \overrightarrow{i-j}\right)\right)$.

4. Обратное вейвлет-преобразование. Обратное вейвлет-преобразование задается двумя фильтрами $h, g \in \mathbb{R}^{\mathbb{Z}}[1]$. Пусть $a, d \in \mathbb{R}^{n}$, тогда компоненты вектора $u \in \mathbb{R}^{2 n}$ задаются следующим образом:

$$
u_{p}=\sum_{n=-\infty}^{+\infty} h_{p-2 n} a_{\{n\}}+\sum_{n=-\infty}^{+\infty} g_{p-2 n} d_{\{n\}} .
$$

Введем обозначения: $u_{i}^{\text {even }}=u_{2 i}, u_{i}^{\text {odd }}=u_{2 i+1}$. Тогда $u_{p}^{\text {even }}=\left(h^{\text {even }} \star a\right)_{p}+$ $\left(g^{\text {even }} \star d\right)_{p}, u_{p}^{\text {odd }}=\left(h^{\text {odd }} \star a\right)_{p}+\left(g^{\text {odd }} \star d\right)_{p}$. Отсюда следует, что преобразование $(3)$ сводится к сумме сверток. Поэтому применимы результаты, полученные в п. 3.

Хотя имеется алгоритм вычисления, в общем случае формула для $\operatorname{SupRows}(A \overrightarrow{i-j})$ громоздка. Однако в случае произвольных фильтров $h$ и $g$, имеющих конкретные $r, l$, можно записать компактную формулу явно. На практике (см. стандарт JPEG2000: ISO/IEC fdc-15444-1, JPEG2000 image coding system) чаще встречается следующий случай - биортогональное вейвлет-преобразование, определяемое фильтрами $h$ и $g$, заданными таблицей

\begin{tabular}{|c|rrrcc|}
\hline$i$ & \multicolumn{1}{|c}{-1} & 0 & +1 & +2 & +3 \\
\hline$h_{i}$ & 0.500 & 1.000 & 0.500 & 0 & 0 \\
$g_{i}$ & -0.125 & -0.250 & 0.750 & -0.250 & -0.125 \\
\hline
\end{tabular}

Применив формулы из п. 3 к векторам $h^{\text {even }}, h^{\text {odd }}, g^{\text {even }}, g^{\text {odd }}$, получаем следующее предложение, из которого вытекает главный результат заметки.

ПредЛОЖениЕ. Пусть $h$ и $g$ - фильтры, описанные в приведенной выше таблице. Tогда $S\left(h^{\text {even }}, \overrightarrow{i-j}\right)=i, E\left(h^{\text {even }}, \overrightarrow{i-j}\right)=j, S\left(h^{\text {odd }}, \overrightarrow{i-j}\right)=i, E\left(h^{\text {odd }}, \overrightarrow{i-j}\right)=$ $j+1, S\left(g^{\text {even }}, \overrightarrow{i-j}\right)=S\left(g^{\text {odd }}, \overrightarrow{i-j}\right)=i-1, E\left(g^{\text {odd }}, \overrightarrow{i-j}\right)=j+1, E\left(g^{\text {even }}, 0\right)=1$, $E\left(g^{\text {even }}, \overrightarrow{i-j}\right)=j$.

СледСтвиЕ. В условиях предложения выполнены равенства: $S(h, \overrightarrow{i-j})=\left\lfloor\frac{i}{2}\right\rfloor$, $S(g, \overrightarrow{i-j})=\left\lfloor\frac{i}{2}\right\rfloor-1, E(g, 0)=1, E(h, \overrightarrow{i-j})=E(g, \overrightarrow{i-j})=\left\lfloor\frac{j+1}{2}\right\rfloor$.

ЗАмечАниЕ. Эту идею можно развить дальше в случае композиции обратных вейвлет-преобразований. Например, вектор $а$ вычисляется аналогичным образом по векторам $a_{1}$ и $d_{1}$. Тем самым мы находим ограничение на множества индексов векторов $a_{1}, d_{1}$ и $d$.

\section{Список литературы}

[1] S. Mallat, A wavelet tour of signal processing, Academic Press, San Diego, 1999.

А. В. Михалёв (А. V. Mikhalev)

Московский государственный университет им. М. В. Ломоносова

E-mail: mikhalev@mech.math.msu.su
Представлено А. В. Михалёвым Принято редколлегией 22.05.2007

\section{А. В. Шокуров (A. V. Shokurov)}

Московский государственный университет им. М. В. Ломоносова

E-mail: anton@fit.com.ru 